\title{
Sirtuin 1 activation enhances the PGC-1 $\alpha$ /mitochondrial antioxidant system pathway in status epilepticus
}

\author{
SHENG-JUN WANG ${ }^{1 *}$, XIU-HE ZHAO ${ }^{1 *}$, WEN CHEN $^{2}$, NING BO $^{3}$, \\ XIAN-JIN WANG ${ }^{4},{\mathrm{ZHAO}-F U ~ \mathrm{CHI}^{1}}^{1}$ and WEI WU ${ }^{1}$ \\ ${ }^{1}$ Department of Neurology, Qilu Hospital, Shandong University, Jinan, Shandong 250012; \\ ${ }^{2}$ Department of Neurology, Jinan Central Hospital, Affiliated to Shandong University, Jinan, Shandong 250013; \\ ${ }^{3}$ Clinical Laboratory, People's Hospital of Shenzhen, Guangdong 518020; ${ }^{4}$ Department of Neurology, \\ Shandong Ankang Hospital, Jining, Shandong 272051, P.R. China
}

Received November 22, 2013; Accepted June 19, 2014

DOI: $10.3892 / \mathrm{mmr} .2014 .2724$

\begin{abstract}
Sirtuin 1 (SIRT1) regulates numerous neuronal processes, including metabolism, antioxidation and aging, through activation of peroxisome proliferator-activated receptor coactivator $1-\alpha$ (PGC-1 $\alpha)$, an upstream regulator of mitochondrial biogenesis and function. However, the role of SIRT1 in the oxidative stress induced by seizures has yet to be elucidated. The present study aimed to investigate whether SIRT1 was involved in the activation of the PGC- $1 \alpha /$ mitochondrial antioxidant system following status epilepticus (SE) in rats. The data demonstrated that SIRT1 expression and activity were enhanced in the rat hippocampus following SE. SIRT1 inhibition effectively blocked the SE-associated increase in PGC-1 $\alpha$ and mitochondrial antioxidant enzymes, including superoxide dismutase 2 (SOD2) and uncoupling protein 2 (UCP2). Additionally, it was also demonstrated that the activation of SIRT1 enhanced mitochondrial electron transport chain complex I activity and increased ATP content. In conclusion, the present results suggest that SIRT1 activation may alleviate mitochondrial oxidative stress induced by seizures partially via PGC-1 $\alpha$ signaling.
\end{abstract}

\section{Introduction}

The major function of mitochondria in cells is the production of cellular energy, ATP, by the mitochondrial respiratory chain. In addition to energy production, the mitochondria is also a

Correspondence to: Dr Wei Wu, Department of Neurology, Qilu Hospital, Shandong University, 107 Wen Hua Xi Road, Jinan, Shandong 250012, P.R. China

E-mail: qiluwuwei71@163.com

\section{*Contributed equally}

Key words: sirtuin, status epilepticus, peroxisome proliferator-activated receptor coactivator $1-\alpha$, superoxide dismutase 2 , uncoupling protein 2 crucial site of reactive oxygen species (ROS) formation. The accumulation of ROS has an important role in the development of neurological disorders, including epilepsy $(1,2)$. The mitochondrial electron transport chain complex enzymes are major sites of ROS generation (3). Our previous studies demonstrated that mitochondrial electron transport chain complex I function was exacerbated following status epilepticus (SE; is a state of persistent seizures, which can result in neuronal damage in the brain), which was attenuated by peroxisome proliferator-activated receptor coactivator $1-\alpha$ (PGC- $1 \alpha$ ) signaling pathway (4). PGC-1 $\alpha$ has been characterized as a major regulator of glucose homeostasis, lipid catabolism, mitochondrial biogenesis and mitochondrial functions (5). PGC- $1 \alpha$ induces the increase of mitochondrial numbers and intracellular ATP concentration in a variety of cells (6). PGC-1 $\alpha$ also suppresses ROS production in cells through the induction of ROS detoxifying enzymes. It has been demonstrated that decreased PGC-1 $\alpha$ expression increases oxidative stress and neurodegeneration (7). It has also been reported that PGC- $1 \alpha$ is required in neurons for the induction of mitochondria related ROS-detoxifying proteins, uncoupling protein 2 (UCP2) and superoxide dismutase 2 (SOD2) (8-10). In a previous study, it was demonstrated that the PGC-1 $\alpha$ signaling pathway was associated with the regulation of the mitochondrial antioxidant system, which could alleviate the oxidative stress damages induced by SE (4).

SIRT1 is one of the mammalian sirtuins, a group of $\mathrm{NAD}^{+}$-dependent deacetylases, which regulate a wide variety of cellular processes, including metabolism, development, cell survival and aging (11). In neurons, SIRT1 prevents mitochondria loss and modulates DNA damage responses (11). The activators of SIRT1 may increase mitochondrial function and energy expenditure (12). More importantly, SIRT1 has been demonstrated to interact directly with PGC-1 to increase PGC-1 $\alpha$ expression and mitochondria biogenesis (13).

The exact roles of SIRT1 on neuron mitochondria functions, particularly the mitochondria antioxidant defense system following SE, remain to be determined. The present study examined the SIRT1 expression in rat hippocampi following SE. In addition, the regulation of SIRT1 on PGC- $1 \alpha / \mathrm{mito}-$ chondria antioxidant proteins in a rodent epileptic model was investigated. 


\section{Materials and methods}

Animals and treatment. Adult male Wistar rats (Experimental Animal Center of Shandong University, Shandong, China) weighing 250-300 g were used. The experimental procedure conformed to the local guidelines of the animal care and use committees of Shandong University (Shandong, China), which were in accordance with the international standards (NIH Publication no.80-23). All efforts were made to minimize animal suffering. The rats were administered $1 \mathrm{mg} / \mathrm{kg}$ scopolamine (subcutaneously; Sigma, St. Louis, MO, USA) prior to $300 \mathrm{mg} / \mathrm{kg}$ pilocarpine (intraperitoneally; Sigma) to prevent peripheral cholinergic effects. Following pilocarpine treatment, the seizure behavior was observed for up to $90 \mathrm{~min}$. The seizure behavior was graded according to a modified Racine scale (14). Only the rats with sustained generalized motor seizures (stage 4 or 5 ) were included in the study. The seizures were terminated by an intraperitoneal injection of $10 \mathrm{mg} / \mathrm{kg}$ diazepam (Sigma) after $60 \mathrm{~min}$. The SIRT1 activator, resveratrol (40 mg/kg intraperitoneally; Sigma) or SIRT1 inhibitor, sirtinol (20 $\mu \mathrm{g} / \mathrm{kg}$ intracerebroventricully; Sigma); was administered $30 \mathrm{~min}$ prior to intraperitoneal injection of pilocarpine.

To examine the SIRT1 expression, mitochondrial electron transport chain complex I activity and ATP content, the rats were divided into five groups: The control and the 3, 8, 24 and $72 \mathrm{~h}$ following SE groups. To investigate the regulation of SIRT1 on the mitochondria antioxidant system and the histological changes in the hippocampi, the rats were divided into four groups: Control, SE, SE + resveratrol and SE + sirtinol groups. The rats were sacrificed by decapitation at 8 or $24 \mathrm{~h}$ following SE. Then hippocampi were quickly removed on an ice-cooled board and stored at $-80^{\circ} \mathrm{C}$. The rats in the control group were only injected with equal volumes of dissolvent. Each experimental group consisted of eight rats.

RNA isolation and quantitative polymerase chain reaction $(q P C R)$. RNA isolation from the snap-frozen whole hippocampi involved use of TRIzol reagent (Invitrogen, Carlsbad, CA, USA). RNA was reverse transcribed by use of M-MLV reverse transcriptase (Fermentas, Glen Burnie, MD, USA). The reaction was conducted in the Light Cycler PCR system (Roche Diagnostics, Mannheim, Germany). The primers were used as follows: Forward: 5'-TCACCACCGAAATCCTTA-3'; and reverse: 5'-GGTGTCTGTAGTGGCTTGAT-3' for PGC- $1 \alpha$; forward: 5'-ACCGAGGAGAAGTACCACGA-3' and reverse: 5'-TAGGGCTCAGGTTTGTCCAG-3' for SOD2; forward: 5'-AATGACCTGTTCTTTGAGGCTGAC-3' and reverse: 5'-GCTTCGACAGTGCTCTGGTA-3' for UCP2; and forward: 5'-TGCTGGTGCTGAGTATGTCGTG-3' and reverse: 5'-CGGAGATGATGACCCTTTTGG-3' for GAPDH. The standard curves for each gene were generated by a serial dilution of RNA isolated from the control rats. The values of the target genes were normalized using the value of the housekeeping gene GAPDH.

Western blot analysis. The hippocampi tissues were homogenized in lysis buffer and centrifuged at $12,000 \mathrm{x} \mathrm{g}$ at $4^{\circ} \mathrm{C}$ for $10 \mathrm{~min}$. The supernatants were collected as cell proteins. The concentration of protein was determined using a bicinchoninic acid protein assay kit (Pierce Biotechnology, Inc., Rockford,
IL, USA). A total of $40 \mu \mathrm{g}$ of protein were separated by sodium dodecyl sulfate-polyacrylamide gel electrophoresis then transferred to nitrocellulose membranes. Following blocking in Tris-buffered saline with Tween-20, the membranes were incubated with a mouse monoclonal primary antibody against SIRT1, PGC-1 $\alpha$, SOD2, UCP2 (1:1000 dilution; Cell Signaling Technology, Inc., Danvers, MA, USA), and $\beta$-actin (1:4000; Santa Cruz Biotechnology, Inc., Santa Cruz, CA, USA) at $4^{\circ} \mathrm{C}$ overnight. Next the membranes were washed and incubated with horseradish peroxidase-conjugated goat anti-rabbit secondary antibody (1:3000; Santa Cruz Biotechnology, Inc.) for $1 \mathrm{~h}$. The immunoreactivity was enhanced by a chemiluminescence kit (Pierce Biotechnology, Inc.) and exposed to film (Fuji, Tokyo, Japan). The band intensity was analyzed with an image analyzer (Alpha Innotech 2200; Alpha Innotech, San Leandro, CA, USA).

Measurement of SIRT1 activity. The SIRT1 activity was measured as described in the SIRT1 Fluorimetric Activity assay/Drug Discovery kit (Biomol Research Laboratories, Inc., Plymouth Meeting, PA, USA). Briefly, an acetylated peptide fragment derived from p53, known to be deacetylated by SIRT1, fluoresces upon deacetylation. Recombinant SIRT1 was preincubated with potential activators or inhibitors of the enzyme for $10 \mathrm{~min}$. The acetylated p53-based substrate was then added and the reaction was allowed to proceed for $45 \mathrm{~min}$. The reaction was quenched by the addition of nicotinamide and the fluorescence was measured uaing Varioskan Flash instrument (Thermo Fisher Scientific, Waltham, MA, USA).

Measurement of mitochondrial electron transport chain complex I activity. The method was conducted as previously described (4). Briefly, mitochondrial protein was isolated from the whole hippocampi. The rotenone-sensitive nicotinamide adenine dinucleotide diaphorase to $\mathrm{CoQ} 1$ oxidoreductase (complex I) and citrate synthase activities were measured spectrophotometrically (Sigma). All of the assays were processed by a thermostatically regulated UV-visible spectrophotometer (Thermo Fisher Scientific).

Measurement of ATP concentration. ATP was measured via an ATP Bioluminescence Assay kit (Roche Diagnostics) according to the manufacturer's instructions. Briefly, hippocampi were washed and lysed on ice with lysis buffer. The homogenates were immediately centrifuged at $14,000 \mathrm{x} \mathrm{g}$ for $5 \mathrm{~min}$ at $4^{\circ} \mathrm{C}$. The supernatants were collected and combined with an equal quantity of luciferase reagent, and the samples were imaged immediately using an Alpha Innotech imaging system (Alpha Innotech).

Histology. The rats were intracardially perfused with $4 \%$ paraformaldehyde at $24 \mathrm{~h}$ following SE. The fixed brain tissue blocks were paraffinized and sectioned coronally at $10 \mu \mathrm{m}$ and Nissl staining with Toluidine Blue was performed. Under a high magnification (x400), the number of surviving pyramidal cells in the hippocampal CA3 region was blindly counted.

Statistical analysis. The values are expressed as the mean \pm standard deviation. Statistical analysis of the results was performed by one-way analysis of variance followed by 

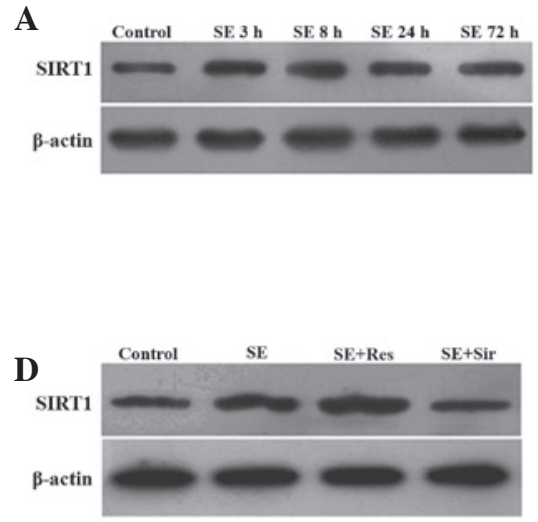

B 4

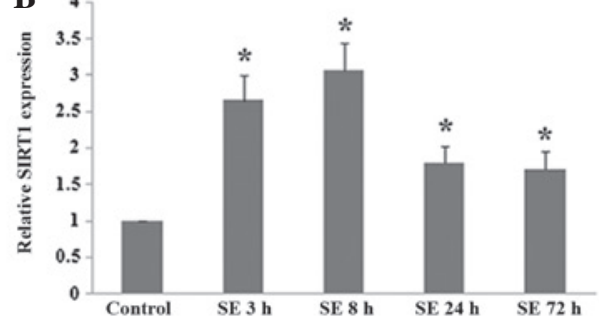

$\mathbf{E}$

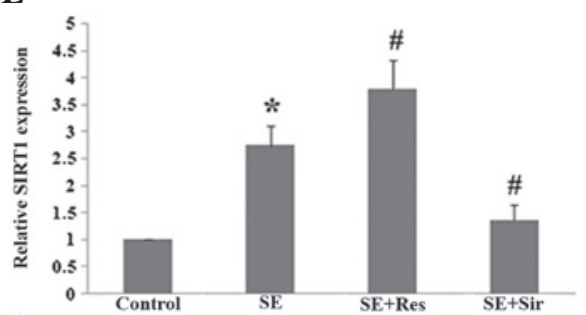

C

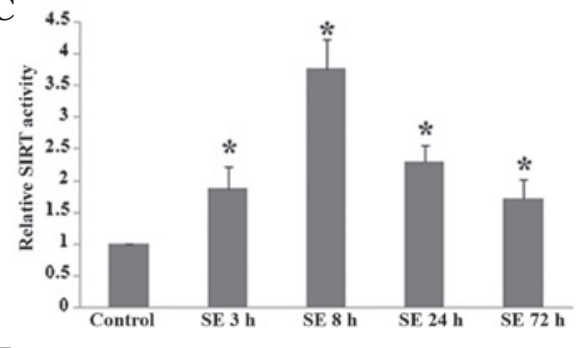

F

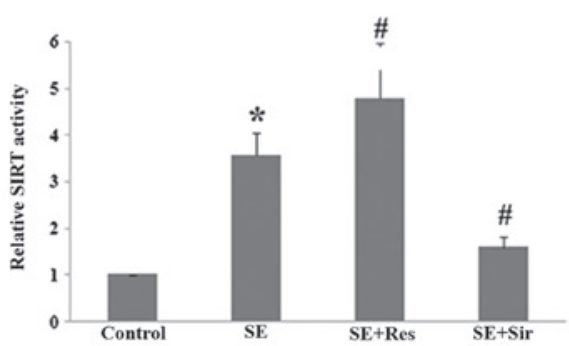

Figure 1. SIRT1 activation in the rat hippocampus following SE. (A) Immunoblotting analysis of the protein expression of SIRT1 at various time points in the rat hippocampus following SE. (B) Quantitative representation of the protein expression of SIRT1 in the rat hippocampus following SE. (C) Quantitative representation of the SIRT1 activity at various time points in rat hippocampus following SE. (D) Immunoblotting analysis of Res or Sir on the protein expression of SIRT1 in the rat hippocampus $8 \mathrm{~h}$ following SE. (E) Quantitative representation of Res or Sir on the protein expression of SIRT1 in the rat hippocampus following SE. (F) Quantitative representation of SIRT1 activity in the rat hippocampus $8 \mathrm{~h}$ following treatment with SE with resveratrol or sirtinol. The data are expressed as the mean \pm standard deviation of seven independent animals. ${ }^{*} \mathrm{P}<0.05$, vs. the control group; ${ }^{*} \mathrm{P}<0.05$, vs. the SE group. SIRT1, sirtuin $1 ;$ SE, status epilepticus; Res, resveratrol; Sir, sirtinol.
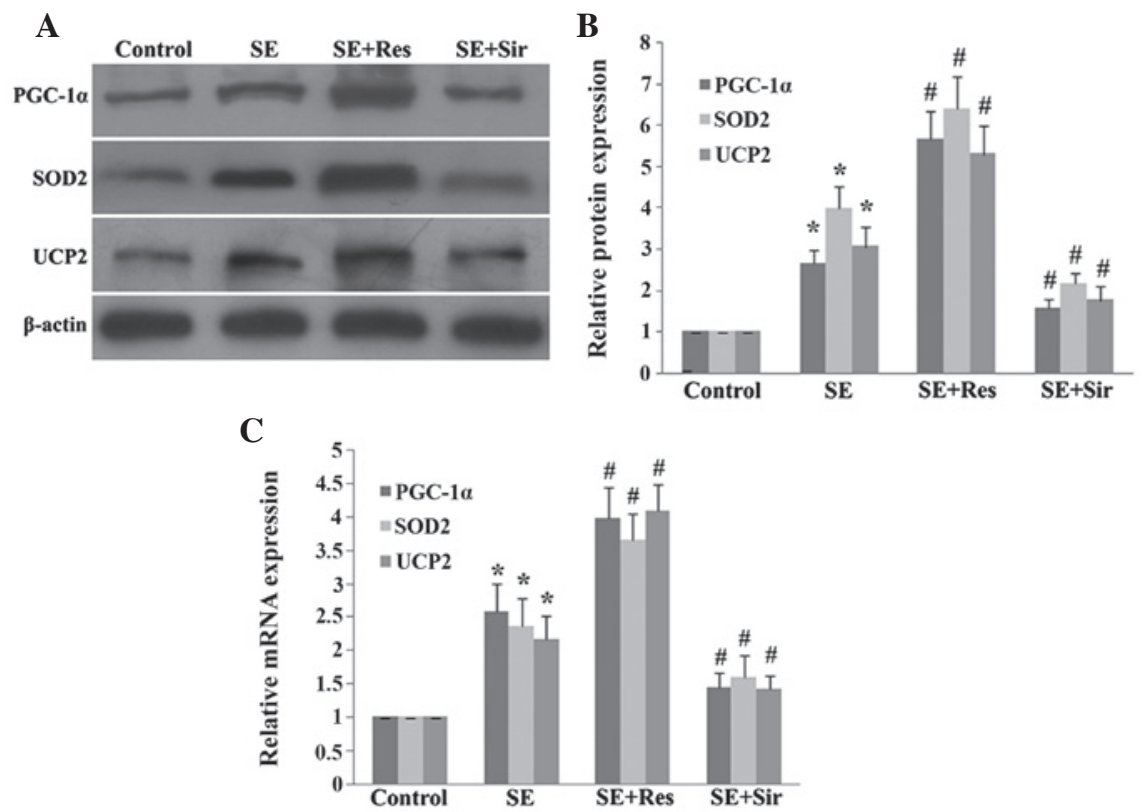

Figure 2. SIRT1 regulates levels of PGC-1 $\alpha$, SOD2 and UCP2 $8 \mathrm{~h}$ following SE in the rat hippocampus. (A) Immunoblotting analysis of resveratrol or sirtinol pretreatment on the protein expression of PGC-1 $\alpha$, SOD2 and UCP2. (B) Quantitative representation of Res or Sir pretreatment on the protein expression of PGC-1 $\alpha$, SOD2 and UCP2 in the rat hippocampus. (C) Quantitative representation of Res or Sir pretreatment on the mRNA expression of PGC-1 $\alpha$, SOD2 and UCP2 in the rat hippocampus following SE. The data are expressed as the mean \pm standard deviation of six independent animals. ${ }^{*} \mathrm{P}<0.05$, vs. the control group; ${ }^{~} \mathrm{P}<0.05$, vs. the SE group. SIRT1, sirtuin 1; PGC-1 $\alpha$, peroxisome proliferator-activated receptor (PPAR) coactivator-1 $\alpha$; SOD2, superoxide dismutase 2; UCP2, uncoupling protein 2; SE, status epilepticus; Res, resveratrol; Sir, sirtinol.

the Newman-Keuls test. $\mathrm{P}<0.05$ was considered to indicate a statistically significant difference.

\section{Results}

Seizure behavior of experimental rats. Seizure behavior induced by pilocarpine followed the typical course of seizures, with a gradual increase in intensity to SE (stage 4 or 5). There was no difference of the percent incidence or onset latency of seizure behavior in the resveratrol or sirtinol pretreatment groups compared with that of the SE group.

SE activates SIRT1 in the rat hippocampus. The protein level of SIRT1 and its activity were examined in the rat hippocampus at different time points following SE. As demonstrated in Fig. 1, the protein level and activity of SIRT1 began to increase at $3 \mathrm{~h}$, 
A

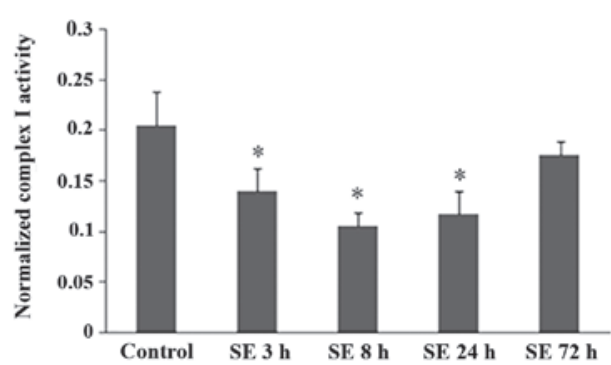

C

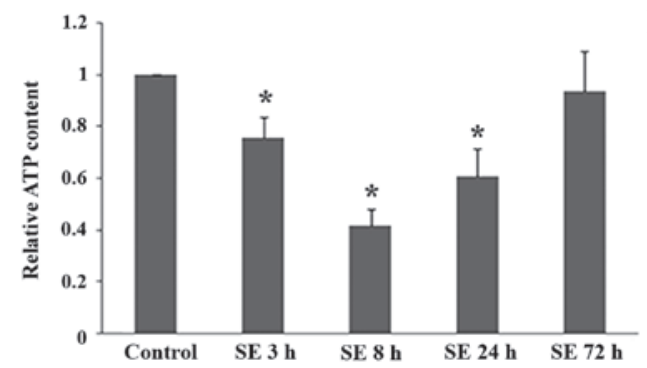

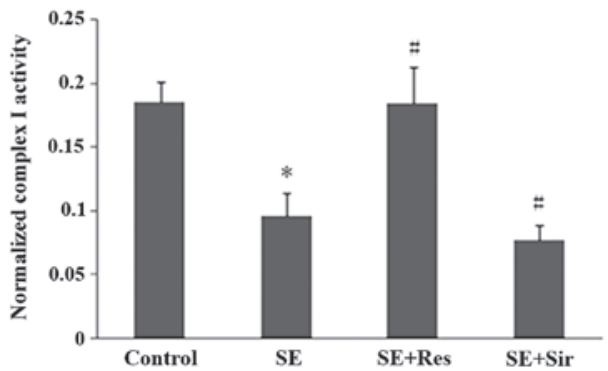

D

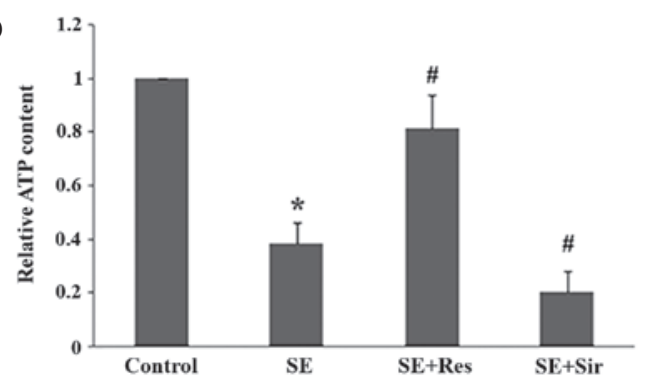

Figure 3. SIRT1 regulates mitochondrial electron transport chain complex I activity and ATP content in the rat hippocampus following SE. (A) Quantitative representation of normalized mitochondrial electron transport chain complex I activity at various time points in the rat hippocampus following SE. (B) Quantitative representation of Res or Sir pretreatment on normalized mitochondrial electron transport chain complex I activity in the rat hippocampus $8 \mathrm{~h}$ following SE. (C) Quantitative representation of ATP content at various time points in the rat hippocampus following SE. (D) Quantitative representation of Res or Sir pretreatment on ATP content in the rat hippocampus $8 \mathrm{~h}$ following SE. The data are expressed as the mean \pm standard deviation and are expressed as folds vs. control of seven independent animals. " $\mathrm{P}<0.05$, vs. the control group; ${ }^{\prime} \mathrm{P}<0.05$, vs. the SE group. SIRT1, sirtuin 1 ; SE, status epilepticus; Res, resveratrol; Sir, sirtinol.

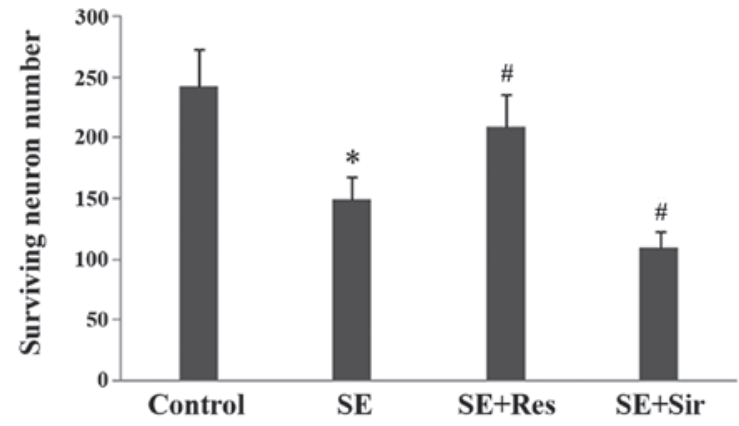

Figure 4. SIRT1 activation alleviates neuron damage in the rat hippocampus following SE. Quantitative representation of surviving pyramidal cell numbers in the CA3 subfield of the hippocampus following SE. The data are expressed as the mean \pm standard deviation of six independent animals. ${ }^{*} \mathrm{P}<0.05$, vs. the control group; ${ }^{\text {"P }} \mathrm{P}<0.05$, vs. the SE group. SIRT1, sirtuin 1; SE, status epilepticus; Res, resveratrol; Sir, sirtinol.

reached a peak at $8 \mathrm{~h}$ and remained elevated $72 \mathrm{~h}$ following $\mathrm{SE}(\mathrm{P}<0.05$; Fig. 1A-C). Resveratrol significantly promoted the expression and activity of SIRT1 at $8 \mathrm{~h}$ following SE $(\mathrm{P}<0.05)$. Additionally, sirtinol effectively inhibited the increase in the protein level and activity of SIRT1 in the rat hippocampus following $\mathrm{SE}(\mathrm{P}<0.05$; Fig. 1D-F).

SIRT1 regulates $P G C-1 \alpha /$ mitochondrial antioxidant enzymes following $S E$. The present study then further examined the effects of SIRT1 on the expression of PGC-1 $\alpha$, SOD2 and UCP2, which are important mitochondrial related ROS-detoxifying enzymes. The protein and mRNA expression of PGC-1 $\alpha$, SOD2 and UCP2 significantly increased in the rat hippocampus at $8 \mathrm{~h}$ following pilocarpine-induced SE induced $(\mathrm{P}<0.05)$. When resveratrol had been administered, the expression of PGC-1 $\alpha$, SOD2 and UCP2 were evidently enhanced $(\mathrm{P}<0.05)$. Consistently, sirtinol significantly suppressed the mRNA or protein overexpression of PGC-1 $\alpha$, SOD2 and UCP2 following SE ( $<<0.05$; Fig. 2).

SIRT1 activation enhances mitochondria functions following $S E$. The mitochondrial electron transport chain complex I activity and ATP content began to decline at $3 \mathrm{~h}$, reached the lowest levels at $8 \mathrm{~h}$ and remained decreased at $24 \mathrm{~h}$ in the rat hippocampal tissues following SE ( $\mathrm{P}<0.05$; Fig. $3 \mathrm{~A}$ and $\mathrm{C})$. As demonstrated in Fig. 3B and D, resveratrol significantly attenuated the suppression of mitochondrial electron transport chain complex I activity and ATP content induced by SE $(\mathrm{P}<0.05)$. Furthermore, after sirtinol was administered, the suppression of mitochondrial electron transport chain complex I activity and ATP content was significantly exacerbated $(\mathrm{P}<0.05)$.

SIRT1 attenuates neuron damage following SE. The present data demonstrated evident pyramidal neuron damage in the CA3 region of the rat hippocampus at $24 \mathrm{~h}$ following SE. The number of surviving neurons was significantly higher in the resveratrol pretreatment group as compared with the SE group ( $\mathrm{n}=6 ; \mathrm{P}<0.05 ;$ Fig. 4). By contrast, sirtinol further aggravated the neuron damage induced by SE.

\section{Discussion}

In the present study, it was demonstrated that SIRT1 protein expression and activity increased in the rat hippocampus at the acute phase of pilocarpine-induced seizures. SIRT1, a class III histone deacetylase, is an important member of the 
sirtuins family. SIRT1 is known to have numerous important functions, including stress protection and metabolism regulation. The results of the present study suggest that SIRT1 may also be a positive regulator of the mitochondrial antioxidant system following the development of seizures. Previous studies demonstrated that SIRT1 activator resveratrol had antioxidant and anti-inflammatory effects (15). Recently, we also demonstrated that resveratrol attenuated the inflammatory responses induced by seizures (16). Resveratrol may reduce neurodegeneration in the rodent hippocampus by the PGC-1 $\alpha$ signaling pathway (17). PGC- $1 \alpha$ is known to be a positive regulator of mitochondrial function and oxidative metabolism (6). Increased PGC-1 $\alpha$ expression accelerates the recovery of mitochondrial and cellular functions following acute cell injury $(6,7)$. PGC-1 $\alpha$ null mice are more sensitive to the neurodegenerative effects of oxidative stress (7). PGC-1 $\alpha$ expression is under the control of several signaling pathways involving nitric oxide, calcium-dependent protein kinases, calcineurin $\mathrm{A}$, adenosine monophosphate-activated protein kinase and p38 MAPK $(6,18,19)$. Previously, it was demonstrated that nitric oxide and AMPK regulate the PGC-1 $\alpha$ and mitochondrial antioxidant system in epileptic rats (4). The present study demonstrated that SIRT1 was also an important upstream regulator of PGC- $1 \alpha$ signaling in seizures induced by pilocarpine. SIRT1 is able to deacetylate specific lysine residues on PGC-1 $\alpha$, mediating gluconeogenesis and mitochondrial biogenesis (13).

PGC-1 $\alpha$ may not only stimulate mitochondrial biogenesis, but may also protect neurons from oxidative injury through the induction of several ROS-detoxifying enzymes $(7,8,20)$. UCP2 and SOD2 are two important ROS-detoxifying mitochondrial proteins (21). SOD2 has been demonstrated to have protective effects against oxidative stress-induced neuronal damage in various experimental models $(22,23)$. UCP2 is able to eliminate free radicals and elevate ATP levels in neurons $(24,25)$. The upregulation of UCP2 decreases the release of ROS and reduces neuronal loss in the brain tissue following ischemic or epileptic injury $(4,24,26)$. Besides regulating the PGC-1 $\alpha$ pathway, SIRT1 is reported to repress mitochondrial UCP2 transcription by binding directly to its promoter (27). Our previous data demonstrated that the levels of UCP2 and SOD2 are increased following SE in association with PGC-1 $\alpha$ signaling (4). The present study revealed that the expression of PGC- $1 \alpha$ and UCP2/SOD2 were upregulated by the SIRT1 activator, resveratrol, in the rat hippocampus following seizures. This indicates that SIRT1 may regulate UCP2 and SOD2, at least partially, via PGC-1 $\alpha$ signaling.

A previous study also suggested that PGC-1 $\alpha$ may activate components in the mitochondrial electron transport chain, including ATP synthase and SOD2 (28). The present data revealed that the SIRT1 activator also upregulated mitochondrial electron transport chain complex I activity and mitochondrial ATP production in rat hippocampi following seizures. These results indicate that SIRT1 is possibly involved in the protection of mitochondrial energy metabolism damage by oxidative stress induced by SE. SE may induce oxidative stress, which leads to neuronal cell death. It was previously demonstrated that SE caused significant neuronal cell death and a marked increase in oxidative stress (4). In the present study, it was identified that the neuron death in the hippocampus of rats following SE was also alleviated by the activator of SIRT1.

In conclusion, the present study demonstrated that SIRT1 is activated in the rat hippocampus following SE. SIRT1 activation enhanced the expression of mitochondrial antioxidant enzymes UCP2/SOD2 and attenuated oxidative stress as revealed by increased levels of ATP and mitochondrial electron transport chain complex I following SE. Furthermore, SIRT1 may be a positive regulator of the mitochondrial antioxidant system following pilocarpine-induced SE, at least in part by regulating PGC-1 $\alpha$. This may assist in understanding the role of mitochondria during epilepsy and provide new targets for developing new antiepileptic drugs.

\section{Acknowledgements}

The present study was supported by grants from National Nature Science Foundation of China (grant no. 81000557) and the Independent Innovation Foundation of Shandong University (grant no. 2012TS145).

\section{References}

1. Balaban RS, Nemoto S and Finkel T: Mitochondria, oxidants, and aging. Cell 120: 483-495, 2005.

2. Waldbaum S and Patel M: Mitochondrial dysfunction and oxidative stress: a contributing link to acquired epilepsy? J Bioenerg Biomembr 42: 449-455, 2010.

3. Dröse S and Brandt U: Molecular mechanisms of superoxide production by the mitochondrial respiratory chain. Adv Exp Med Biol 748: 145-169, 2012

4. Han YX, Lin YT, Xu JJ, et al: Status epilepticus stimulates peroxisome proliferator-activated receptor $\gamma$ coactivator $1-\alpha /$ mitochondrial antioxidant system pathway by a nitric oxide-dependent mechanism. Neuroscience 186: 128-134, 2011.

5. Wu Z and Boss O: Targeting PGC-1 alpha to control energy homeostasis. Expert Opin Ther Targets 11: 1329-1338, 2007.

6. Rasbach KA and Schnellmann RG: PGC-1alpha over-expression promotes recovery from mitochondrial dysfunction and cell injury. Biochem Biophys Res Commun 355: 734-739, 2007.

7. St-Pierre J, Drori S, Uldry M, Silvaggi JM and Rhee J: Suppression of reactive oxygen species and neurodegeneration by the PGC-1 transcriptional coactivators. Cell 127: 397-408, 2006.

8. Valle I, Alvarez-Barrientos A, Arza E, Lamas S and Monsalve M: PGC-1 $\alpha$ lpha regulates the mitochondrial antioxidant defense system in vascular endothelial cells. Cardiovasc. Res 66: 562-573, 2005.

9. Csiszar A, Labinskyy N, Pinto JT, et al: Resveratrol induces mitochondrial biogenesis in endothelial cells. Am J Physiol Heart Circ Physiol 297: H13-H20, 2009.

10. Sun AY, Wang Q, Simonyi A and Sun GY: Resveratrol as a therapeutic agent for neurodegenerative diseases. Mol Neurobiol 41: 375-383, 2010.

11. Houtkooper RH, Pirinen E and Auwerx J: Sirtuins as regulators of metabolism and healthspan. Nat Rev Mol Cell Biol 13: 225-238, 2012.

12. Della-Morte D, Dave KR, DeFazio RA, et al: Resveratrol pretreatment protects rat brain from cerebral ischemic damage via a sirtuin 1-uncoupling protein 2 pathway. Neuroscience 159: 993-1002, 2009.

13. Nemoto S, Fergusson MM and Finkel T: SIRT1 functionally interacts with the metabolic regulator and transcriptional coactivator PGC-1\{alpha\}. J Biol Chem 280: 16456-16460, 2005.

14. Racine RJ: Modification of seizure activity by electrical stimulation. II. Motor seizure. Electroencephalogr Clin Neurophysiol 32: 281-294, 1972.

15. Udenigwe CC, Ramprasath VR, Aluko RE and Jones PJ: Potential of resveratrol in anticancer and anti-inflammatory therapy. Nutr Rev 66: 445-454, 2008.

16. Wang SJ, Bo QY, Zhao XH, et al: Resveratrol pre-treatment reduces early inflammatory responses induced by status epilepticus via mTOR signaling. Brain Res 1492: 122-129, 2013. 
17. Kim D, Nguyen MD, Dobbin MM, et al: SIRT1 deacetylase protects against neurodegeneration in models for Alzheimer's disease and amyotrophic lateral sclerosis. EMBO J 26: 3169-3179, 2007.

18. Cantó C and Auwerx J: PGC-1alpha, SIRT1 and AMPK, an energy sensing network that controls energy expenditure. Curr Opin Lipidol 20: 98-105, 2009.

19. Rowe GC, Jiang A and Arany Z: PGC-1 coactivators in cardiac development and disease. Circ Res 107: 825-838, 2010.

20. Chen SD, Lin TK, Yang DI, et al: Protective effects of peroxisome proliferator-activated receptors gamma coactivator-1alpha against neuronal cell death in the hippocampal CA1 subfield after transient global ischemia. J Neurosci Res 88: 605-613, 2010.

21. Rubiolo JA,Mithieux G and Vega FV: Resveratrol protects primary rat hepatocytes against oxidative stress damage: activation of the Nrf 2 transcription factor and augmented activities of antioxidant enzymes. Eur J Pharmacol 591: 66-72, 2008.

22. Vincent AM, Russell JW, Sullivan KA, et al: SOD2 protects neurons from injury in cell culture and animal models of diabetic neuropathy. Exp Neurol 208: 216-227, 2007.
23. Fukui M and Zhu BT: Mitochondrial superoxide dismutase SOD2, but not cytosolic SOD1, plays a critical role in protection against glutamate-induced oxidative stress and cell death in HT22 neuronal cells. Free Radic Biol Med 48: 821-830, 2010

24. Andrews ZB, Horvath B, Barnstable CJ, et al: Uncoupling protein-2 is critical for nigral dopamine cell survival in a mouse model of Parkinson's disease. J Neurosci 25: 184-191, 2005.

25. Ho PW, Ho JW, Liu HF, et al: Mitochondrial neuronal uncoupling proteins: a target for potential disease-modification in Parkinson's disease. Transl Neurodegener 1: 3, 2012.

26. Mehta SL and Li PA: Neuroprotective role of mitochondrial uncoupling protein 2 in cerebral stroke. J Cereb Blood Flow Metab 29: 1069-1078, 2009

27. Bordone L, Motta MC, Picard F, et al: Sirt1 regulates insulin secretion by repressing UCP2 in pancreatic beta cells. PLoS Biol 4: e31, 2006.

28. Kong X, Wang R, Xue Y, et al: Sirtuin 3, a new target of PGC-1alpha, plays an important role in the suppression of ROS and mitochondrial biogenesis. PLoS One 5: e11707, 2010. 